\title{
One-step split GFP staining for sensitive protein detection and localization in mammalian cells
}

\author{
Lara Kaddoum ${ }^{1,3}$, Eddy Magdeleine ${ }^{1,3}$, Geoffrey S. Waldo ${ }^{4}$, Etienne Joly ${ }^{1,3}$, and Stéphanie Cabantous ${ }^{2,3}$ \\ ${ }^{1}$ CNRS, Institute of Pharmacology and Structural Biology (IPBS), Toulouse, France, ${ }^{2}$ INSERM U563, \\ Département Innovation Thérapeutique et Oncologie Moléculaire, Institut Claudius Régaud, Université Paul \\ Sabatier, Toulouse, France, ${ }^{3}$ Université Paul Sabatier, IPBS, Toulouse, France, and ${ }^{4}$ Bioscience Division, MS-M888, \\ Los Alamos National Laboratory, Los Alamos, NM, USA
}

BioTechniques 49:727-736 (October 2010) doi 10.2144/000113512

Keywords: epitope tagging; protein localization; split GFP; green fluorescent protein; intracellular staining; protein fragment complementation

Supplementary material for this article is available at www.BioTechniques.com/article/113512.

Although epitope tags are useful to detect intracellular proteins and follow their localization with antibodies, background and nonspecific staining often remain problematic. We describe a simple assay based on the split GFP complementation system. Proteins tagged with the 15-amino acid GFP 11 fragment are detected with a solution of the recombinant nonfluorescent complementary GFP 1-10 fragment to reconstitute a fluorescent GFP. In contrast to antibody-based staining methods, this one-step assay presents high specificity and very low background of fluorescence, thus conferring higher signal-to-noise ratios. We demonstrate that this new application of the split GFP tagging system facilitates detection of proteins displaying various subcellular localizations using flow cytometry and microscopy analysis.

Understanding protein dynamics in living cells requires sensitive and quantitative tools for measuring spatiotemporal modifications of protein expression and/or localization. Fusion to green fluorescent protein (GFP) or its derivatives enables direct visualization of intracellular proteins without the need for secondary reagents or treatment of the cell (1). However, the large size of GFP may alter protein localization and behavior (2), and the permanent fluorescence of the reporter can be a hindrance to detect other markers. To detect native intracellular proteins, monoclonal or polyclonal antibodies are sometimes available, but they need to recognize an epitope conserved after fixation. Alternatively, various epitope tags (myc, His, HA, Flag) have been developed to specifically detect or isolate proteins in cells (3). Detection of such tags either involves specific monoclonal antibodies directly linked to a fluorochrome or successive steps of unlabeled antibody and fluorochrome-conjugated secondary reagents (4). Such protocols are often timeconsuming, as they require several washing steps to remove all unbound reagents between binding reactions. Moreover, low signal-tonoise ratios are often observed, due to the presence of nonspecifically bound antibodies, especially for polyclonal antibodies, or due to endogenous expression of the epitope in the parent cell (5).

As a possible alternative, we have investigated whether we could use the split GFP tagging system for the intracellular detection of proteins (6), which is based on the auto-assembly capacity of two nonfluorescent portions of GFP-GFP 1-10 and GFP 11-to restore a fully fluorescent GFP. The GFP 11 tag, which is only 15 amino acids long, is fused to the $\mathrm{N}$ or $\mathrm{C}$ terminus of the coding sequence of the protein of interest and can then be expressed in eukaryotic cells. The GFP 1-10 detector fragment is produced separately in Escherichia coli and purified from inclusion bodies as previously described (7). After fixation and permeabilization of cells expressing the GFP 11-tagged protein, the refolded GFP $1-10$ protein is added in trans, allowing the two split GFP fragments to associate spontaneously and restore the GFP fluorescence (Figure 1A). Here, we describe the application of the split GFP protein complementation assay for detecting GFP 11-tagged proteins in mammalian cells relative to antibody staining using FACS and microscopy analysis.

\section{Materials and methods}

Plasmids

pcDNA 3.1 vector expressing human MeCP2e1-Myc-His was provided by Dr. Berge A. Minassian of the Hospital for Sick Children, Toronto, Ontario, Canada (8). For FK506 binding protein 12 and $\mathrm{MeCP} 2 \mathrm{e} 1$, the respective coding sequences were inserted at the $\mathrm{N}$ terminus of GFP 11 , in a vector derived from pEGFP_N3 (Clontech Laboratories, Saint-Germain-en-Laye, France) (see sequence of the mammalian GFP 11 cassette below). To generate GFP 11-H-Ras, full-length GFP was replaced by the 15-amino acid mammalian GFP 11 peptide (GFP11m) in the pEGFP-H-Ras plasmid kindly provided by J. LippincotSchwartz (9).

DNA sequence of the GFP11m

vector cassette

GFP11 m N-terminal fusion. 5'-ATGGGCCGGGACCACATGGTGCTGCACGAGTACGTGAACGCCGCCGGCATCACAGACGGCGGCAGCGGCGGCGGCAGC-3'.

GFP11m C-terminal fusion. 5'-GGCGACGGCGGCAGCGGCG- 
A
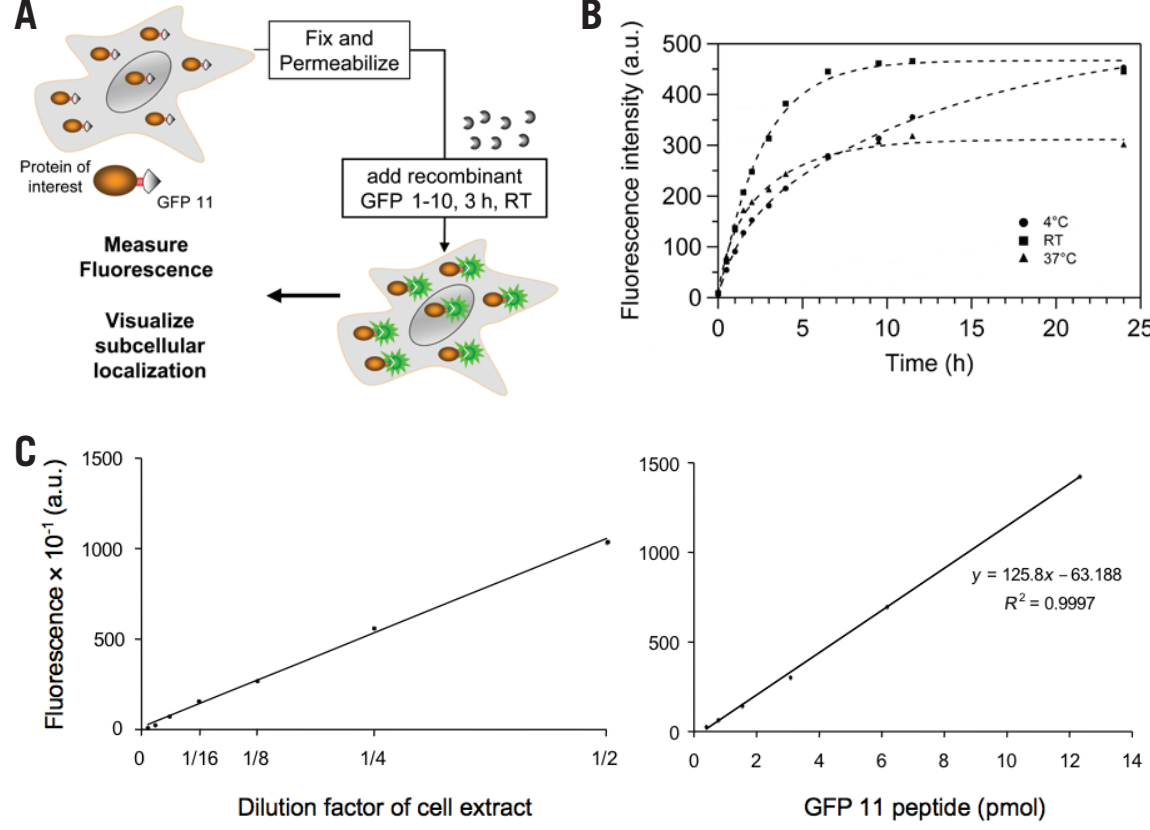

Figure 1. Characterization of the GFP 1-10 in vitro assay in mammalian cells. (A) Cells expressing GFP 11-tagged protein are fixed and permeabilized. Reconstituted GFP 1-10 protein solution is added and incubated at RT for $3 \mathrm{~h}$. Fluorescence is measured and compared with control cells. (B) Kinetic profiles of complementation reaction of N2A-MeCP2-GFP 11 cells incubated with GFP 1-10 at three temperatures: $4^{\circ} \mathrm{C}(\bullet), 20^{\circ} \mathrm{C}(\boldsymbol{\bullet})$, and $37^{\circ} \mathrm{C}(\boldsymbol{\Delta})$. FACS measurements of fluorescence intensity were performed at various time intervals. (C) Using the GFP 1-10 complementation assay to evaluate the amounts of protein in cell extracts. Cell extracts (left graph) and peptide dilutions (right graph) were complemented with recombinant GFP 1-10 in solution in a 96-well plate, and fluorescence was measured after an overnight incubation at $4^{\circ} \mathrm{C}$. The quantity of GFP 11-tagged protein in cell extract dilutions was then deduced from a standard calibration curve with GFP 11 peptide solution (right graph). a.u., arbitrary units.

GCGGCAGCCGGGACCACATGGTGCTGCACGAGTACGTGAACGCCGCCGGCATCACATAA-3'.

Recombinant GFP 1-10. Expression of GFP 1-10 detection reagent was performed in E. coli BL21 (DE3). Recombinant protein was purified from inclusion bodies as described previously (7). For each set of assays, $37.5 \mathrm{mg}$ purified inclusion bodies were used to prepare $15 \mathrm{~mL}$ GFP 1-10 solution $(2.5 \mathrm{mg} / \mathrm{mL})$ in $50 \mathrm{mM}$ Tris, $\mathrm{pH}$ 7.4, $0.1 \mathrm{M} \mathrm{NaCl}, 10 \%$ glycerol (TNG).

\section{Cell culture}

Neuro2A (N2A) cells were maintained in DMEM supplemented with $10 \%$ FCS, 100 $\mathrm{U} / \mathrm{mL}$ penicillin, $100 \mu \mathrm{g} / \mathrm{mL}$ streptomycin, $10 \mathrm{mM}$ HEPES, $0.1 \mathrm{mM}$ nonessential amino acids, and $1 \mathrm{mM}$ sodium pyruvate. Human embryonic kidney (HEK) 293 cells were grown in RPMI-1640 medium supplemented with $10 \%$ FCS, $100 \mathrm{U} / \mathrm{mL}$ penicillin, $100 \mu \mathrm{g} / \mathrm{mL}$ streptomycin, and $2 \mathrm{mM}$ L-glutamine.

\section{Transfection}

Transfections were carried out using JetPEI (Polyplus Transfection, Illkirch, France) following the manufacturer's instructions. Depending on the resistance gene carried by the plasmid vector, stable clones were selected with either $500 \mu \mathrm{g} / \mathrm{mL}$ G418 (Invitrogen, Cergy Pontoise, France) or $125 \mu \mathrm{g} / \mathrm{mL}$ Zeocin (InvivoGen, Toulouse, France) and thereafter maintained at these concentrations. Monoclonal cell lines were obtained by single cell dilution in 96-well plates. Cells used as negative control were untransfected, and were thereby not cultured in the presence of selecting drugs.

\section{Antibodies}

Primary antibodies. Rabbit polyclonal anti-MeCP2 antibody (crude serum) was generated after immunization of a rabbit with a synthetic peptide corresponding to amino acids 465-478 of mouse MeCP2 (C-PRPNREEPVDSRTP; performed by Millegen, Labège, France). Mouse anti-Myc IgG1 antibody was purified with protein A beads (Pierce, Thermo Scientific, Brebières, France) from clone 9E10 hybridoma supernatants (purchased from ATCC, Manassas, VA, USA). Rat monoclonal anti-H-Ras antibody was purchased from Santa Cruz Biotechnology (Cat. no. sc-35; Santa Cruz, CA, USA).
Secondary antibodies. Alexa Fluor 488-conjugated goat anti-rabbit, Alexa Fluor 647-conjugated goat anti-rabbit, rhodamine-conjugated goat anti-rabbit, Alexa Fluor 488-conjugated goat antimouse, Alexa Fluor 647-conjugated goat anti-rat, and rhodamine-conjugated goat anti-rat were obtained from Molecular Probes, Invitrogen.

Flow cytometry analysis

For each condition, $2 \times 10^{5}$ cells were trypsinized, washed three times with PBS, then fixed with $4 \%$ paraformaldehyde for $10 \mathrm{~min}$ at $4^{\circ} \mathrm{C}$. After two washes with PBS, cells were permeabilized in $0.1 \%$ Triton-X 100 in PBS for 10 min at $4^{\circ} \mathrm{C}$. Cells were then washed twice with 2\% FCS PBS before incubation with the primary antibody (rabbit anti-MeCP2 serum, dilution 1/300, or mouse anti-Myc, dilution $1 / 300$, home-purified solution at $4 \mathrm{mg} / \mathrm{mL}$, or rat anti-H-Ras, dilution $1 / 200$ ) for $45 \mathrm{~min}$ at $4^{\circ} \mathrm{C}$ (dilution in $2 \%$ FCS PBS). After three washes with $2 \%$ FCS PBS, cells were incubated with secondary antibody (dilution 1/200) for $30 \mathrm{~min}$ at $4^{\circ} \mathrm{C}$. Cells were washed again three times with $2 \%$ FCS PBS buffer and then analyzed using a FACS Calibur cytometer and CellQuest software (BD Biosciences, Le Pont de Claix, France).

For GFP 1-10 detection, the recombinant protein solution was diluted to $0.15 \mathrm{mg} / \mathrm{mL}$ in $2 \%$ FCS PBS and directly added to the fixed/permeabilized cells. After incubation at room temperature (RT) for $3 \mathrm{~h}$, fluorescence was analyzed using a FACScalibur flow cytometer. For double staining, cells were labeled with GFP 1-10 for $3 \mathrm{~h}$ at RT, washed twice with $2 \%$ FCS PBS buffer, and then incubated with the appropriate antibodies.

\section{Immunofluorescence staining and microscopy}

N2A cells $\left(1 \times 10^{5}\right.$ cells per well $)$ or HEK 293 cells $\left(1 \times 10^{5}\right.$ cells per well $)$ stably expressing MeCP2, FKBP, or H-Ras tagged with either GFP 11 or GFP were plated overnight on glass coverslips in 24-well plates. Cells were washed twice with PBS and fixed with $3.7 \%$ paraformaldehyde (PFA) in PBS for 30 min at RT. After two washes with PBS, cells were permeabilized in $0.3 \%$ Triton-X 100 for $10 \mathrm{~min}$ at RT. For GFP fusions, cells were washed twice with $2 \%$ FCS PBS, and after a final wash with PBS, coverslips were mounted in 4',6-diamidino2-phenylindole (DAPI)-containing ProLong Gold antifading reagent (Molecular Probes, Invitrogen). For GFP 1-10 detection, GFP 11-expressing 
A
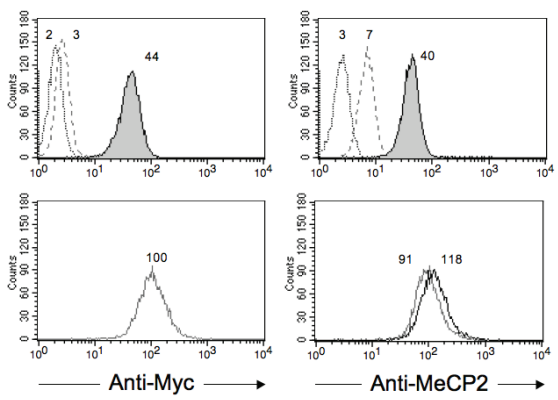

Pre-immune serum for anti-MeCP2 2 taining

lgG control for anti-Myc staining

Secondary antibody for anti-MeCP2 and ant-Myc staining

Without GFP 1-10

C

FKBP-GFP 11

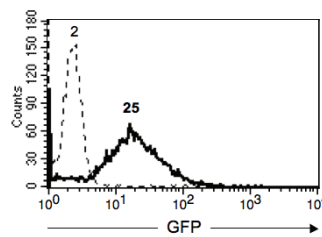

N2A

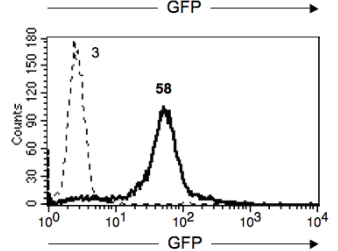

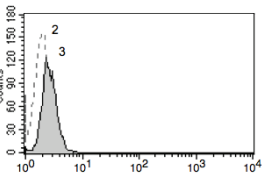

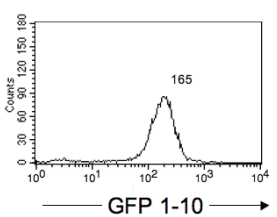

N2A

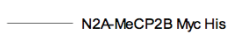

N2A-MeCP2B GFP 11

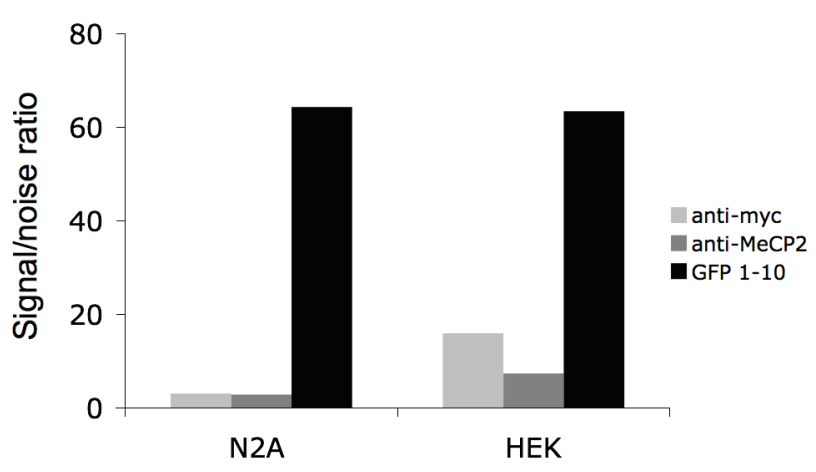

D
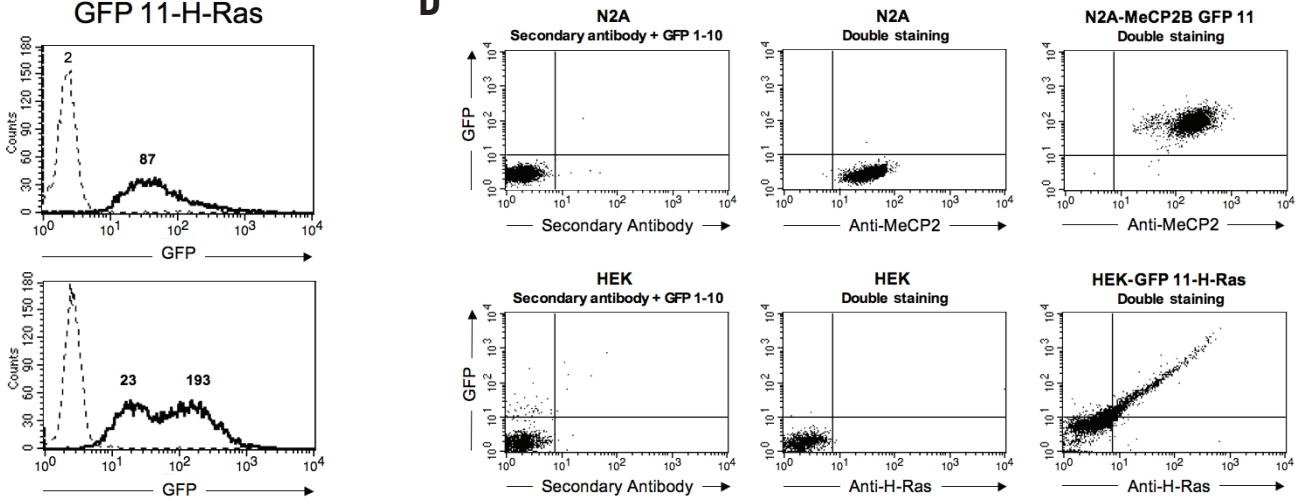

Figure 2. GFP 1-10 staining assay for FACS analysis. (A) Comparison with MAb staining. FACS detection of MeCP2-myc-His and MeCP2-GFP 11 in N2A cells using anti-myc MAb (left), anti-MeCP2 polyclonal (middle), or recombinant GFP 1-10 (right). Negative controls are shown on the top row. Positive signals obtained from transfected N2A cells expressing MeCP2 are shown in the bottom row: MeCP2-myc-His (gray) or MeCP2-GFP 11 (black). (B) Signal-to-noise ratio for FACS detection of MeCP2 using staining with either anti-myc, anti-MeCP2 antibody, or recombinant GFP 1-10, in N2A and HEK cells. (C) FACS detection of FKBP-GFP 11 and GFP 11-H-Ras in N2A and HEK cells. Nontransfected N2A or HEK cells, dashed line; N2A or HEK cells expressing GFP 11-tagged proteins, black. (D) Double staining with antibodies (far red, FL4, $x$ axis) and GFP1-10 (green, FL1, $y$ axis). (Top row) N2A stably expressing MeCP2-GFP 11 or not. (Bottom row) HEK cells transiently transfected with GFP 11-H-Ras or not. Left panel, fluorescence of secondary antibodies on control cells; middle panel, anti-MeCP2 or anti-H-Ras signals on nontransfected control cells; right panel, double staining of transfectants.

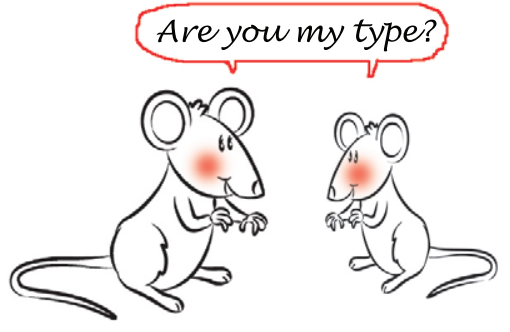

EZ Fast PCR Genotyping Kit

Fast: $\quad$ 10-min DNA extraction

Easy: One solution extraction

Reliable: Strong PCR bands

\section{EZBioResearch}

www.EZBioResearch.com Tel: 800-637-0262 cells were incubated for $3 \mathrm{~h}$ at $\mathrm{RT}$ with GFP 1-10 protein solution diluted to $0.15 \mathrm{mg} / \mathrm{mL}$ in $2 \%$ FCS PBS. Cells were washed with PBS and mounted in DAPIcontaining ProLong Gold antifading reagent. For double staining, cells were labeled with GFP 1-10, washed twice with $2 \%$ FCS PBS and then incubated with primary antibody (rabbit anti-MeCP2 diluted to $1 / 300$ and rat anti-H-Ras diluted to $1 / 200$ ) for $1 \mathrm{~h}$ at RT. After three washes with $2 \%$ FCS PBS, cells were incubated with secondary antibodies (anti-rabbit IgG, rhodamine, and anti-rat IgG, rhodamine, diluted to $1 / 400$ ) for $1 \mathrm{~h}$ at RT. Cells were washed with PBS and mounted in DAPI-containing ProLong Gold antifading reagent. We used a DM-RB fluorescence microscope (Leica, Nanterre, France) with a $40 \times$ and $100 \times$ oil immersion objective to visualize the stained cells. Images were acquired with a CoolSNAP HQ camera (Photometrics, Roper Scientific, Evry, France) and analyzed with MetaMorph (Molecular Devices, Sunnyvale, CA, USA) or ImageJ (http://rsbweb.nih.gov/ij).

Microplate assay

Cells $\left(5 \times 10^{6}\right)$ were trypsinized and washed three times with PBS. The cell pellet was flash-frozen in liquid nitrogen. The frozen pellet was resuspended in 40 $\mu \mathrm{L}$ extraction buffer ( $25 \%$ glycerol, 0.42 $\mathrm{M} \mathrm{NaCl}, 1.5 \mathrm{mM} \mathrm{MgCl}, 0.2 \mathrm{mM}$ EDTA, 20 mM HEPES, pH 7.9, 0.5 mM DTT). In a 96-well fluorescence plate, $50 \mu \mathrm{L}$ total cell extracts were serial diluted in 100 $\mu \mathrm{L}$ final volume of TNG buffer. These dilutions $(50 \mu \mathrm{L})$ were mixed with 100 $\mu \mathrm{L} 0.3 \mathrm{mg} / \mathrm{mL}$ GFP $1-10$ protein solution. The plate was incubated overnight at $4^{\circ} \mathrm{C}$. Fluorescence was measured using a FLx800 Fluorescence Microplate Reader (BioTek, Colmar, France). For titration curves, starting concentrations of GFP 11 peptide $(0.03 \mathrm{mg} / \mathrm{mL})$ were used and diluted serially in $50 \mu \mathrm{L}$ TNG buffer. 
A

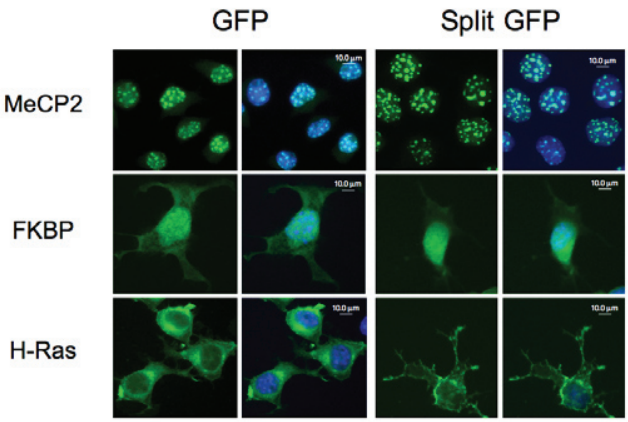

C
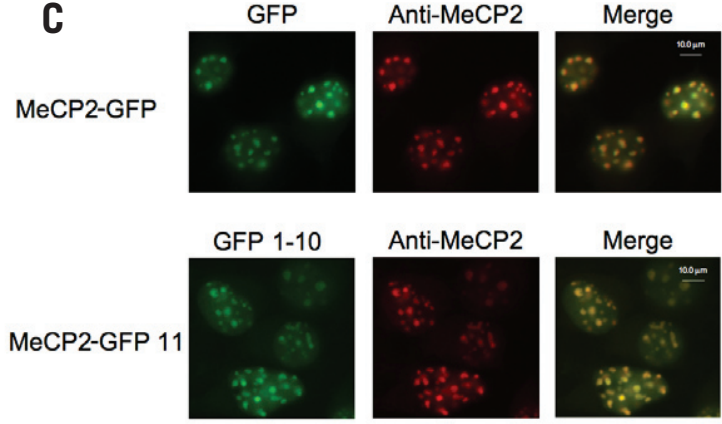
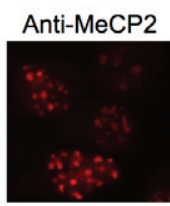

Merge

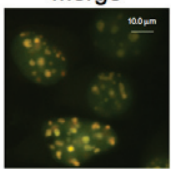

B
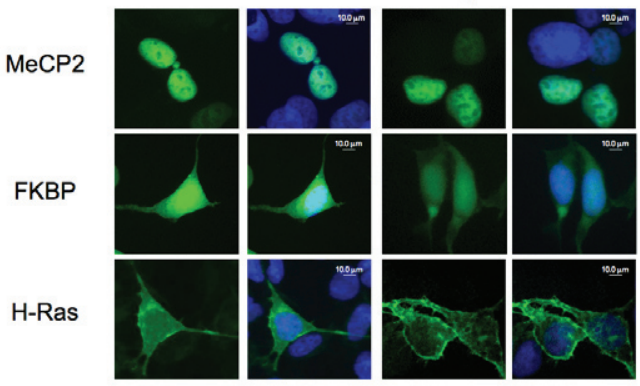

Figure 3. GFP 1-10 staining for studying protein localization by fluorescence microscopy. Three proteins-MeCP2, FKBP, and $H$-Ras- were expressed in ( $\mathrm{A}$ ) N2A cells or (B) HEK 293 cells, as GFP fusions (left panels) or GFP 11 fusions (right panels). MeCP2-GFP 11, FKBP-GFP 11, and GFP 11-H-Ras were stained with recombinant GFP 1-10 reagent before mounting on microscope slides. Left image, green fluorescence at 488-nm excitation. Right image, overlay of green fluorescence and DAPI nuclear staining (blue). Scale bars, $10 \mu \mathrm{m}$. Double staining experiments are shown in panels $\mathrm{C}$ and D. Fluorescein isothiocyanate (FITC) emission channel at $530 \mathrm{~nm}$ with excitation at $488 \mathrm{~nm}$ (left), rhodamine emission channel detected at $590 \mathrm{~nm}$ with excitation at $545 \mathrm{~nm}$ (middle), superimposition of both images (right). (C) Anti-MeCP2 sera and GFP 1-10 staining were performed on N2A-MeCP2-GFP 11 cells and compared with N2A cells expressing MeCP2-GFP fusions. (D) Anti-H-Ras MAb and GFP 1-10 staining on HEK-GFP 11-H-Ras and GFP-H-Ras HEK cells.

\section{Results and discussion}

Characterization of the GFP 1-10 staining assay for FACS

We initially developed this method for the detection of the nuclear methyl-CpGbinding protein 2 (hMeCP2el) in mammalian cells (8). The results shown in Figures 1 and 2 were obtained with a monoclonal cell line that expresses homogenous levels of MeCP2-GFP 11, as detected with an anti-MeCP2 polyclonal antibody. Cells in suspension were fixed in $4 \%$ PFA, permeabilized with $0.1 \%$ Triton, and then incubated in a solution of $0.3 \mathrm{mg} / \mathrm{mL} \mathrm{GFP} 1-10$ protein at $4^{\circ}, 20^{\circ}$, and $37^{\circ} \mathrm{C}$. Fluorescence increase was followed by flow cytometry (FACS) at different time intervals for $24 \mathrm{~h}$. Kinetic plots demonstrated a first order reaction with temperature-dependent rates (Figure 1B). A slower kinetic of complementation was observed at $4^{\circ} \mathrm{C}$, reaching a plateau after overnight incubation (Figure $1 \mathrm{~B}$ and Supplementary Table $\mathrm{S} 1$ ). At $37^{\circ} \mathrm{C}$, a faster reaction rate was observed, but final fluorescence value was considerably reduced, probably because of unfolding of the renatured GFP 1-10 protein. Our optimal condition was obtained at RT, in which maximum fluorescence was reached after $12 \mathrm{~h}$, similarly to what had been observed previously in E. coli (6). Background fluorescence, determined by

\section{Future of BIOTECH OPTICS.}

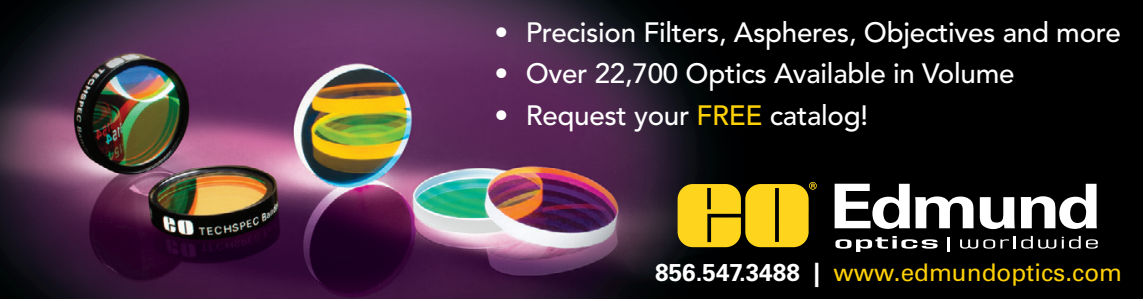

incubating nontransfected N2A cells with GFP 1-10 was very low, but increased slightly over time, and the best signal-to-noise ratios, in excess of 130, were thus attained after 3-4 h of staining (Supplementary Table S1).

Sensitivity of the GFP 1-10 staining in microplate assay

To evaluate the sensitivity of the split GFP assay with eukaryotic cell extracts, we performed the complementation in 96-well plates. N2A cells $\left(6 \times 10^{6}\right)$ expressing MeCP2-GFP 11 were used to obtain a final volume of $100 \mu \mathrm{L}$ total cell extract. Serial 2-fold dilutions of the sample were performed in $50 \mu \mathrm{L}$ TNG buffer and then incubated with GFP $1-10$ at $4^{\circ} \mathrm{C}$. Fluorescence was measured on a microplate reader after overnight incubation. As a control, cell extract from N2A cells was also measured in the same dilution range and was substracted from the signal. As observed previously with bacterially produced proteins (7), sensitivity plots showed an excellent linear relationship between fluorescence and protein quantity (Figure $1 \mathrm{C}$, left). To evaluate protein concentration of MeCP2-GFP 11, a standard titration curve was performed using GFP 11 peptide (Figure 1C, right). From this, we evaluated 


\section{Easy Way}

\author{
for
}

\section{Your Life Science Research!}

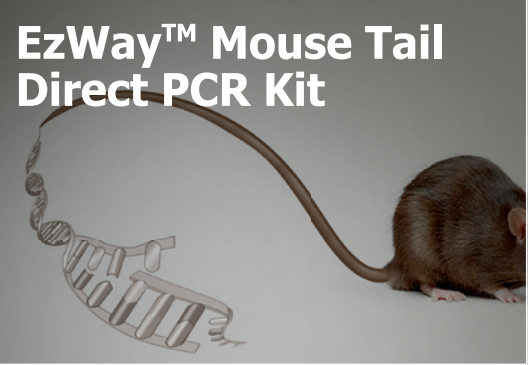

True rapid and simple method for genotyping in transgenic mouse without DNA extraction

- No DNA Purification

- No Use of Proteinase K

- No Overnight incubation

- Just a 10-minute lysis

\section{pink-ONE Cytokine ELISA Kit}

Stop to worry about pipetting error throughout whole steps of ELISA assay

- pink colored Assay Diluent and TMB

- Easy tracking of reagent addition during pipetting

- No intereference with enzyme development

- Broad cytokines detection ranges from human, mouse, rat

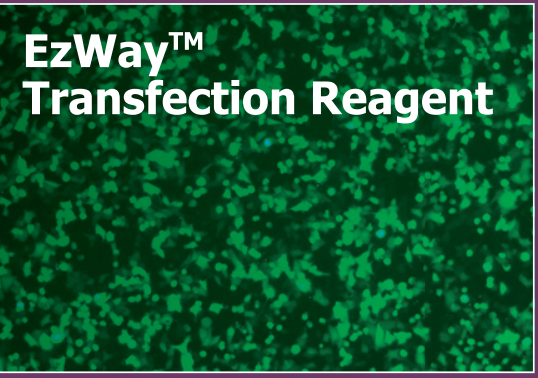

High efficiency in transfection with minimal cytotoxicity in multiple cell types

- Low Cellular Toxicity

- High Transfection Efficiency

- Efficient siRNA \& DNA delivery

- Ready-to-use reagent that this particular clone of $\mathrm{N} 2 \mathrm{~A}$ transfectants contained, on average, $1.5 \times 10^{6}$ copies of GFP 11-tagged MeCP2 protein of the same order of magnitude as levels reported in physiological conditions (10). The limit of sensitivity of the plate-based assay was 0.5 pmol GFP 11 peptide, as previously observed with bacterially produced proteins (6), and this corresponded to a $1 / 100$ dilution of the starting cellular extract ( $8 \mu \mathrm{g}$ total protein) (Figure 1C). These results demonstrate that the linearity and the sensitivity of the assay are suited for monitoring protein expression in mammalian cells.

\section{Comparison with MAb staining}

Using FACS, we then compared the sensitivity of the split GFP detection system with antibody staining (5) (Figure 2A). We used another clone of $\mathrm{N} 2 \mathrm{~A}$ cells transfected with a vector coding for MeCP2 tagged with the myc-His double epitope, which expresses protein levels similarly to the MeCP2-GFP 11 clone, as detected by anti-MeCP2 antibody (Figure 2A, bottom row, middle panel). As expected with polyclonal antibodies, staining with the rabbit pre-immune serum gave some background compared with cells reacted solely with the secondary anti-rabbit antibody. Staining with immune anti-MeCP2 serum resulted in a higher signal than the pre-immune serum from the same rabbit, although undifferentiated N2A cells do not express detectable levels of endogenous MeCP2 protein (Reference 11 and our own observations). Detection of the myc epitope using the 9E10 MAb (mouse anti-myc; ATCC) gave higher levels of staining than its isotype control on N2A control cells, presumably because of the binding of the antibody to the endogenous myc protein (Figure 2A, top left panel). Nevertheless, specific staining with anti-myc or anti-MeCP2 on N2A cells transfected with the MeCP2-myc-His vector resulted in 3- to 5-fold higher signal values relative to nontransfected N2A cells (Figure 2A). With the GFP 1-10 assay, no detectable difference was observed in fluorescence intensities between unstained and stained N2A control cells (Figure 2A, top right panel). In cells expressing MeCP2-GFP 11, fluorescence signal was clearly separated from the negative control, resulting in signal-to-noise ratios $>60$ (Figure 2B). Similar results were obtained after stable expression of the same constructs in HEK 293 cells (Supplementary Figure $\mathrm{S} 1$ and Figure 2B).

\section{Validation of the GFP 1-10 staining} assay for FACS measurements To further document the versatility of this system, we fused the GFP 11 tag to the N terminus of the H-Ras oncogene protein [localized mostly at the plasma membrane (12)] and to the $C$ terminus of the nucleocytosolic immunophilin FK506 binding protein 12 (FKBP) (13). We established stable populations for these constructs in $\mathrm{N} 2 \mathrm{~A}$ and HEK cells and stained them using GFP 1-10 protein for FACS analysis. For both proteins tagged with GFP 11, although the levels of expression were considerably lower than for MeCP2, the very low levels of background fluorescence of GFP 1-10 on nontransfected cells resulted in wellseparated peaks (Figure 2C). To verify that the split GFP method can be combined with antibody staining, we then performed double staining on N2A-MeCP2-GFP 11 and HEK 293-GFP 11-H-Ras cells. The procedure involved a first step using the GFP 1-10 assay, followed by immunostaining using rhodamine-conjugated antibodies (see the "Materials and methods" section). FACS analysis allowed the detection of both epitopes in the green (GFP) and red (anti-MeCP2 or anti-H-Ras) fluorescence (Figure 2D). Similar levels of detection were obtained for GFP 11 and MeCP2 (Figure 2D, top right graph), suggesting a good sensitivity of the split GFP method. Moreover, a clear correlation was observed between monoclonal anti-H-Ras Ab detection and GFP 1-10 staining for HEK cells transiently transfected with GFP $11-\mathrm{H}-\mathrm{R}$ as, in which only $25 \%$ of the cell population expressed significant levels of the GFP 11-H-Ras protein (Figure 2D). We noticed that background on control cells with monoclonal anti-H-Ras antibody was very low (Figure 2D, middle panel). Even in these conditions, although the difference was less striking than for the anti-myc and anti-MeCP2 antibodies presented above, we found that GFP 1-10 staining of transiently transfected HEK cells still yielded better signal-to-noise ratios than antibody staining (50 versus 13) (Supplementary Figure S2).

\section{GFP 1-10 staining for microscopy}

We next compared cellular localization of the three proteins tagged either with full-length GFP or with GFP 11 (Figure 3). In N2A cells stably expressing either MeCP2-GFP or MeCP2-GFP 11, fluorescence revealed a typical punctuated nuclear pattern seen in murine cells, due to the binding of $\mathrm{MeCP} 2$ to heterochromatin foci (14) (Figure 3A, top row). In HEK cells, heterochromatin is less condensed, and fluorescence was thus diffused throughout the nuclei (15) (Figure 3B, top row). The FKBP protein is generally distributed through the nucleus and the cytosol, but may be predominantly nuclear when it dimerizes (16). In N2A and HEK cells, both FKBP-GFP and FKBP-GFP 11 
fusions were found mostly localized in the perinuclear region (Figure 3, A and B, middle rows). Finally, both GFP-H-Ras and GFP 11-H-Ras fusions were found mostly associated with the plasma membrane. For GFP-H-Ras however, we noticed the presence of additional intracellular vesicular structures in both cell types [Figure 3, A and B (lower rows) and D]. This observation is reminiscent of previous reports showing that fusion of $\mathrm{H}-\mathrm{R}$ as to the whole GFP molecule resulted in its redistribution toward the ER and Golgi membrane, whereas unmodified H-Ras was found mostly at the plasma membrane (17). Our results thereby suggest that the smaller size of the GFP 11 tag may alter the natural distribution of the tagged protein less than full-length GFP. To confirm that staining with split GFP is nonperturbing and correlates accurately with true protein localization, we performed double staining experiments, using specific antibodies against MeCP2 and H-Ras followed by rhodamine-coupled secondary antibody, for both GFP 11-tagged and GFP-tagged fusions of $\mathrm{MeCP} 2$ and $\mathrm{H}-\mathrm{R}$ as; this resulted in perfect co-localization of the red and green signals (Figure 3, C and D). Importantly, for both MeCP2 and H-Ras fusions, no background fluorescence was observed in the other channel when only one of the stainings was performed (Supplementary Figures S3 and S4).

The split GFP detection system is particularly well-suited for protein tagging and detection in eukaryotic cells using multiple formats. Expression and localization can be simultaneously performed either in fixed models or in living cells with transient or stable expression of GFP 1-10 (18). The main advantages over existing epitope tags $(19,20)$ are the high specificity and quantitative recognition between GFP 11 and GFP 1-10 fragments and the absence of fluorescence of the GFP 1-10 protein. This confers very low background signals and facilitates staining procedures, as it does not require extensive washing steps compared with classical immunostaining methods. The small size of the GFP 11 fragment (15 amino acids) should be less perturbing than the bulky GFP, and GFP 1-10 staining can be performed in combination with other immunostaining procedures as for GFP. The versatility of the system will be further enhanced by the possibilities of combining the split GFP method with chromatic variants of GFP, such as cyan and yellow 1-10. Engineering red autofluorescent split proteins would expand the color repertoire of the split GFP system to perform simultaneous detections in vitro and ultimately in vivo, for example, in mice expressing the 1-10 fragment ubiquitously. Both possibilities are currently being pursued in our laboratories.

\section{Acknowledgments}

We are grateful to Evert Haanappel for his help with fitting kinetics curves, Christine Bordier for helping with fluorescence microscopy, and Denis Hudrisier and Jean Denis Pédelacq for helpful discussions. This work was supported by Association Française du Syndrome de Rett (AFSR) and Fondation pour la Recherche Medicale (FRM).

\section{Competing interests}

The split GFP and related intellectual properties are the subject of domestic and foreign patent applications by Los Alamos National Laboratories on behalf of the Department of Energy and LANS, L.L.C.

\section{References}

1. Tsien, R.Y. 1998. The green fluorescent protein. Annu. Rev. Biochem. 67:509-544.

2.Hanson, D.A. and S.F. Ziegler. 2004. Fusion of green fluorescent protein to the C-terminus of granulysin alters its intracellular localization in comparison to the native molecule. J. Negat. Results Biomed. 3:2.

3. Brizzard, B. 2008. Epitope tagging. BioTechniques 44:693-695.

4.Krenacs, T., L. Krenacs, and $M$. Raffeld. 1999. Multiple antigen immunostaining procedures. Methods Mol. Biol. 115:223-233.

5. Evan, G.I., G.K. Lewis, G. Ramsay, and J.M. Bishop. 1985. Isolation of monoclonal antibodies specific for human c-myc proto-oncogene product. Mol. Cell. Biol. 5:3610-3616.

6. Cabantous, S., J.D. Pedelacq, B.L. Mark, C. Naranjo, T.C. Terwilliger, and G.S. Waldo. 2005. Recent advances in GFP folding reporter and split-GFP solubility reporter technologies. Application to improving the folding and solubility of recalcitrant proteins from Mycobacterium tuberculosis. J. Struct. Funct. Genomics 6:113-119.

7. Cabantous, S. and G.S. Waldo. 2006. In vivo and in vitro protein solubility assays using split GFP. Nat. Methods 3:845-854.

8. Mnatzakanian, G.N., H. Lohi, I. Munteanu, S.E. Alfred, T. Yamada, P.J. MacLeod, J.R. Jones, S.W. Scherer, et al. 2004. A previously unidentified MECP2 open reading frame defines a new protein isoform relevant to Rett syndrome. Nat. Genet. 36:339-341.

9. Choy, E., V.K. Chiu, J. Silletti, M. Feoktistov, T. Morimoto, D. Michaelson, I.E. Ivanov, and M.R. Philips. 1999. Endomembrane trafficking of ras: the CAAX motif targets proteins to the ER and Golgi. Cell 98:69-80.

10. Skene, P.J., R.S. Illingworth, S. Webb, A.R. Kerr, K.D. James, D.J. Turner, R. Andrews, and A.P. Bird. 2010. Neuronal $\mathrm{MeCP} 2$ is expressed at near histone-octamer levels and globally alters the chromatin state. Mol. Cell 37:457-468.

11. Young, J.I., E.P. Hong, J.C. Castle, J. CrespoBarreto, A.B. Bowman, M.F. Rose, D. Kang, R. Richman, et al. 2005. Regulation of RNA splicing by the methylation-dependent transcriptional repressor methyl-CpG binding protein 2 . Proc. Natl. Acad. Sci. USA 102:17551-17558.

12. Willumsen, B.M., A. Christensen, N.L. Hubbert, A.G. Papageorge, and D.R. Lowy. 1984. The p21 ras C-terminus is required for transformation and membrane association. Nature 310:583-586.

13. Wang, T. and P.K. Donahoe. 2004. The immunophilin FKBP12: a molecular guardian of the TGF-beta family type I receptors. Front. Biosci. 9:619-631.

14. Marchi, M., A. Guarda, A. Bergo, N. Landsberger, C. Kilstrup-Nielsen, G.M. Ratto, and M. Costa. 2007. Spatio-temporal dynamics and localization of MeCP2 and pathological mutants in living cells. Epigenetics 2:187-197.

15. Akbarian, S., R.Z. Chen, J. Gribnau, T.P. Rasmussen, H. Fong, R. Jaenisch, and E.G. Jones. 2001. Expression pattern of the Rett syndrome gene MeCP2 in primate prefrontal cortex. Neurobiol. Dis. 8:784-791.

16. De Angelis, D.A., G. Miesenbock, B.V. Zemelman, and J.E. Rothman. 1998. PRIM: proximity imaging of green fluorescent proteintagged polypeptides. Proc. Natl. Acad. Sci. USA 95:12312-12316.

17. Zheng, H., J. McKay, and J.E. Buss. 2007. H-Ras does not need COP I- or COP II-dependent vesicular transport to reach the plasma membrane. J. Biol. Chem. 282:25760-25768.

18. Van Engelenburg, S.B. and A.E. Palmer. 2010. Imaging type-III secretion reveals dynamics and spatial segregation of Salmonella effectors. Nat. Methods 7:325-330.

19. Shevtsova, Z., J.M. Malik, U. Michel, U. Scholl, M. Bahr, and S. Kugler. 2006. Evaluation of epitope tags for protein detection after in vivo CNS gene transfer. Eur.J. Neurosci. 23:1961-1969.

20. Lobbestael, E., V. Reumers, A. Ibrahimi, K. Paesen, I. Thiry, R. Gijsbers, C. Van den Haute, Z. Debyser, et al. 2010. Immunohistochemical detection of transgene expression in the brain using small epitope tags. BMC Biotechnol. 10:16.

Received 27 May 2010; accepted 2 August 2010.

Address correspondence to Stéphanie Cabantous, INSERM U563, Département Innovation Thérapeutique et Oncologie Moléculaire, Institut Claudius Régaud, Université Paul Sabatier, 24 rue du Pont Saint-Pierre, 31052 Toulouse Cedex, France, e-mail: cabantous.stephanie@claudiusregaud.fr or Etienne Joly, CNRS, Institute of Pharmacology and Structural Biology (IPBS), 205 route de Narbonne, F-31077 Toulouse, France, email: joly@ipbs.fr 